\title{
Matters of Neural Network Repository Designing for Analyzing and Predicting of Spatial Processes
}

\author{
Stanislav A. Yamashkin ${ }^{1}$ \\ Anastasiya A. Kamaeva ${ }^{4}$ \\ Institute of Institute of Electronic and \\ Lighting Engineering \\ National Research Mordovia State \\ University \\ Saransk, Russia
}

\author{
Anatoliy A. Yamashkin ${ }^{2}$ \\ Geography Faculty \\ National Research Mordovia State \\ University \\ Saransk, Russia
}

\author{
Ekaterina O. Yamashkina ${ }^{3}$ \\ Institute of Information Technologies \\ MIREA - Russian Technological \\ University \\ Moscow, Russia
}

\begin{abstract}
The article is devoted to solving the scientific problem of accumulating and systematizing models and machine learning algorithms by developing a repository of deep neural network models for analyzing and predicting of spatial processes in order to support the process of making managerial decisions in the field of ensuring conditions for sustainable development of regions. The issues of architecture development and software implementation of a repository of deep neural network models for spatial data analysis are considered, based on a new ontological model, which makes it possible to systematize models in terms of their application for solving design problems. An ontological model of a deep neural network repository for spatial data analysis is decomposed into the domain of deep machine learning models, problems being solved and data. Special attention is paid to the problems of storing data in the repository and the development of a subsystem for visualizing neural networks using a graph model. The authors have shown that for organizing a repository of deep neural network models, it is advisable to use a scientifically grounded set of database management systems integrated into a multi-model storage, characterizing the domains of using relational and NoSQL storages.
\end{abstract}

Keywords-Repository; deep learning; artificial neural network; spatial data; visual programming

\section{INTRODUCTION}

A significant role in solving the problem of strengthening the connectivity of territories of countries and regions is played by the introduction of effective digital infrastructures of spatial data (SDI), aimed at operational diagnostics of natural-socialproduction systems (NSPS) and high-precision forecasting of the development of natural processes and phenomena [1]. The core of systems of this class is represented by methods and algorithms for machine analysis of spatial data, which allow solving a whole range of applied problems - anomaly detection, data classification, data fusion. The subject of analysis can be space imagery data, aerial photography, information arrays about natural, social and economic objects with a distributed geospatial organization [2]. The areas of application of the results of the analysis in the national economy are extremely wide - from increasing the efficiency of agriculture to assessing the consequences of natural processes $[3,4]$.
SDI as a system can be decomposed into subsystems for storing, analyzing and distributing geospatial data. The development of the federal spatial data infrastructure is necessary in order to effectively solve the problem of remote monitoring of mobile objects and geographically distributed resources in order to ensure information connectivity of countries and create conditions for sustainable development of the country [5]. At present, the functioning of spatial data infrastructures should be based on and application of new effective methods, approaches and algorithms for the analysis of spatio-temporal data, which can function both on the basis of classical hard and soft computations based on the complex application of fuzzy logic, neural network models, evolutionary modeling [6].

With the development of the scientific and engineering basis and computing power, the direction of deep machine learning has recently been strengthened, based on the systematic use of many levels of nonlinear information processing for the extraction and transformation of hierarchical features, analysis and classification of patterns. Despite the growing interest in the topic of deep machine learning, this problem area requires serious study in the direction of strengthening the project orientation of the process of using deep neural network models for analyzing large spatial data [7]. This statement is based on the assumption that the processes of designing complex neural networks, determining their hyperparameters, the format of input and output data, and integrating training data arrays should be determined by the features of real problems to be solved [8]. In other words, the use of deep neural network models for solving practical design problems can be effective if the conditions for the consolidation of accumulated knowledge are met, heuristics and rules are collected into a single system, for interaction with which convenient graphical and application program interfaces are organized. Quality documentation on the use and flexible customization of pretrained models is of key importance. The indicated provisions determine the feasibility of forming a repository of deep neural network models in the digital SDI system, which provides system access to the storage of neural network models, as well as tools for choosing the optimal solution in a specific problem area and disclosing the features of its use. 
The purpose of this article is to discuss problematic issues devoted to solving the problem of machine learning models systematizing by developing a repository of deep neural network models for analyzing and predicting of spatial processes. The matters of architecture development and software implementation of deep neural network models repository are considered.

\section{RELATED WORKS}

The task of designing and training effective deep neural network models for analyzing large arrays of spatial data encounters many problematic moments that require finding solutions. This article is devoted to solving the scientific problem of accumulating and systematizing models and machine learning algorithms by developing a repository of deep neural network models for analyzing and predicting the development of spatial processes in order to support the process of making managerial decisions in the field of ensuring conditions for sustainable development of regions. The purpose of the study is to provide support for the process of making management decisions in the field of forming conditions for sustainable development of regions through the development and development of a repository of deep neural network models for the analysis of spatial data [9]. The solution to the problem of forming the architecture and software implementation of a repository of deep neural network models should be based on a new ontological model that defines a formalized description of the topologies of deep models, tasks to be solved, a set of analyzed data, learning algorithms, as well as relationships between these entities [10].

The development of information systems based on machine learning algorithms is very different from the development of traditional software applications based on specifications and repeatedly tested algorithms. In addition, the process of training machine learning models can be expensive and computationally intensive. This fact determines the feasibility of creating a tool that provides the ability to reuse pre-trained machine learning models for the development of applied applications. The use of pretrained machine learning models deployed as web services is one of the top technology trends. Popular frameworks such as Tensorflow [11] and PyTorch [12] provide high-level API support for using machine learning model components.

Since deploying and maintaining machine learning models is still a non-trivial task that requires in-depth knowledge of machine learning and systems administration, organizations are building repositories of machine learning models in order to simplify the use of models within their business processes. Other companies form repositories that can be accessed by third parties. The Wolfram Neural Net Repository (launched in June 2018) [13] and the AWS marketplace (launched in November 2018) [14] are useful examples. Each model repository has a different structure and uses different heuristics to group the models. For example, AWS labels each model using a set of criteria (including the analyzed data), and each model can meet several criteria. Machine learning model repositories promise to close the operational gap between data scientists and software developers. In the field of spatial data analysis, the task of developing a repository of neural network models has its own specifics.

\section{Methodology And Methods of ReseARCH}

Analysis of the strengths and weaknesses of the currently existing repositories of deep neural network models of general purpose (AWS Marketplace, Open Neural Network Exchange, Wolfram Neural Net Repository and others) [15] made it possible to form a list of specific problems, the solution of which will ensure the creation of an effective system suitable for solving specific practical oriented tasks in the field of spatial data analysis.

1) Designing an ontological model for the storage of deep neural networks.

2) Development of a storage scheme for models of deep machine analysis of spatial data in the form of a metalanguage.

3) Implementation of the function of converting repository models into representations used by modern software systems for machine learning.

4) Development of graphical web interfaces for a repository of deep neural network models with navigation functions.

5) Creation of a user authentication subsystem in order to differentiate access rights to deep neural network models.

6) Development of interfaces for obtaining structured information about specific neural network models.

7) Development of a subsystem for visualizing deep machine learning models in the form of graph-schemes within the framework of adaptive web interfaces.

8) Development of an application programming interface based on the architectural style of REST, to provide a unified program exchange.

9) Updating the repository of deep neural network models in the process of solving practice-oriented tasks in the field of ensuring conditions for sustainable development of Russian regions.

10)Development of a recommendation system for the selection and configuration of deep neural network models.

Work on the design, development and implementation of a repository of deep neural network models involves obtaining results characterized by scientific novelty. So, the ontological model of the storage of deep neural networks should be distinguished by the achievement of a detailed and comprehensive systematization of deep machine learning models used to analyze large spatial data by classes of design problems to be solved, types of analyzed data, architecture, objective (numerical) and subjective (expert) performance metrics [16]. On the basis of the ontological model, a conceptual storage scheme for deep machine analysis models can be created in the form of a meta-language, which allows them to be converted into representations used by modern machine learning frameworks [17].

Graphical web interfaces of the repository of deep neural network models should be justified from the standpoint of system analysis of user experience, allowing the selection of a 
relevant machine learning model for solving specific problems of spatial data analysis, obtaining systematized information about the required deep neural network model. It is also supposed to justify and deploy a subsystem for visualizing deep learning models through web interfaces in the form of a graph diagram, with the function of online editing of the model topology within the capabilities of a thin web client, as well as organizing an application programming interface based on REST architectural style to provide unified programmatic interaction with the repository. Of particular importance is the design and development of a new recommender system for the selection and configuration of deep neural network models, which allows for a relevant search for an effective architectural solution and its fine tuning for solving design problems through a graphical web interface of the neural network repository [18]. The repository of deep neural network models acquires practical value if the database is updated with new models for the analysis of spatial data, including remote sensing data. Each deep neural network should be tested on test sites in order to determine estimates of its effectiveness in solving practical problems: classification of remote sensing data, forecasting the development of natural processes.

\section{RESUlTS AND Discussion}

\section{A. Development of an Ontological Model of a Repository}

The development of a deep neural network repository should be based on an ontology that provides a formalized description of entities (ANN topologies, learning processes and accuracy assessment), as well as the relationships between them [19]. The relevance of this provision is determined by the hypothesis that the process of using deep learning to solve design problems can be supported only if the acquired knowledge, heuristics and rules are collected in a system for which convenient means of interaction are organized.

An ontological model of a deep neural network repository for spatial data analysis can be decomposed into domains of deep machine learning models, tasks to be solved, and data (Fig. 1). This allows you to give a comprehensive definition of the formalized area of knowledge: each stored model must be compared with a set of specific tasks and data sets (tensor, raster, vector, attributive). The domain of machine learning models is defined by concepts and relationships that describe various topologies (branching and chain structures of layers of various types (including fully connected, convolutional, recurrent) with various activation and regularization functions), as well as learning methods and algorithms.

The deep model is characterized by the format of the data received for analysis and the type of the output signal, the loss function, the initialization algorithm, the learning strategy (with a teacher, without a teacher, with partial involvement of a teacher, with reinforcement). Each deep model is associated with a meta description containing its category, characteristics of the project-oriented tasks to be solved, and guidelines for applied use. The design solution must store pre-trained copies, ready for solving design problems after fine-tuning. The software implementation of the repository was carried out on the basis of the artifacts of the object-oriented design stage, including the determination of the use cases of the system, the construction of structural and behavioral UML diagrams. The development of graphical interfaces of the repository must be carried out using UI / UX design methodologies using a software stack of web technologies, which will allow you to use the repository from anywhere in the world connected to the Internet. The API for interacting with external systems is based on the REST architectural style.

Thus, the process of forming a repository of deep neural networks in a digital SDI system should be based on a projectoriented approach, based on which each stored deep ANN should be compared with the range of design tasks within which it can be used.

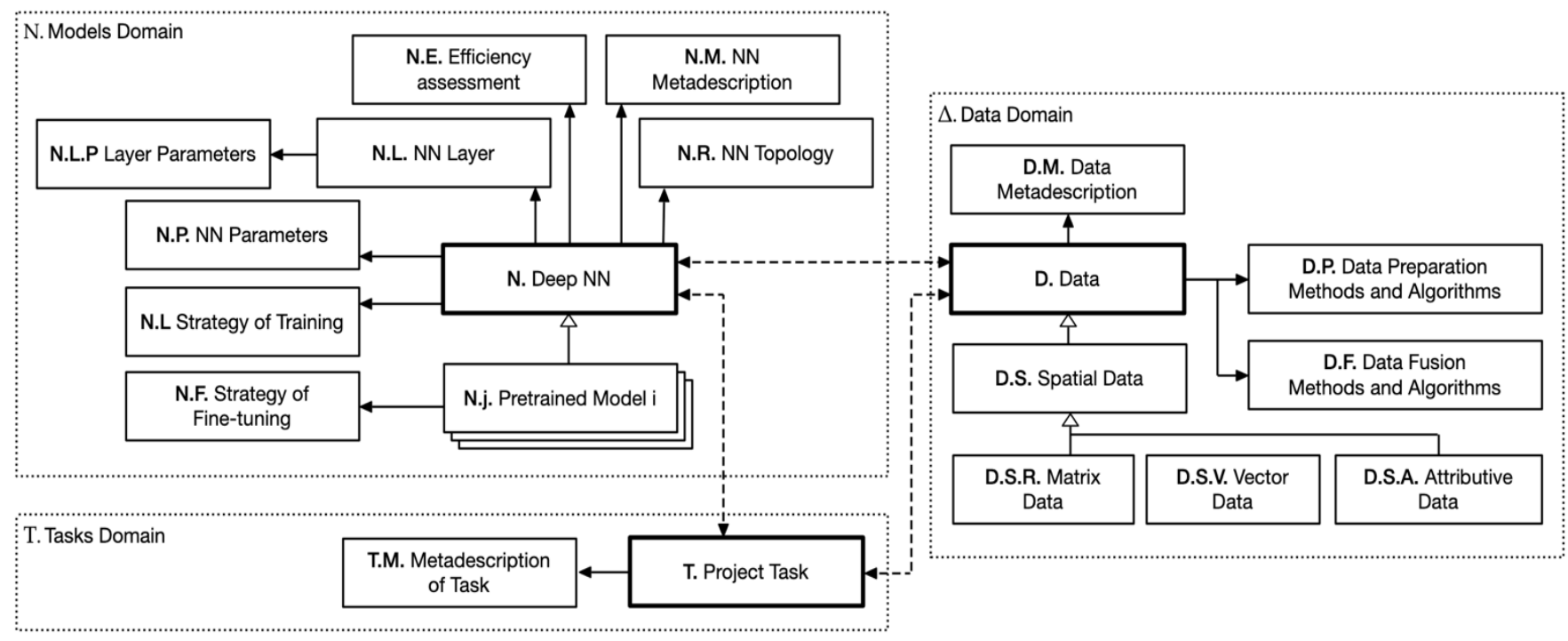

Fig. 1. Ontological Model of the Repository. 


\section{B. Designing the Storage Subsystem of Repository}

To organize a data warehouse of a repository of neural networks, it is advisable to use a comprehensive science-based set of database management systems (DBMS). So, in relational storages, the organization of the data integration process is based on entities and relationships established between them. The highly organized structure and flexibility makes relational storage powerful and adaptable to different types of data domains, tasks, and models. To store spatial data, it is advisable to use a relational DBMS with support for spatial operations: PostgreSQL MySQL. Alternative advantages to the process of integrating spatial data can be provided by NoSQL storages, which should be classified into the following categories: a) resident databases - Redis (preferred for the development of data caching systems, buffers of high-speed exchange with the repository through software interfaces); b) document databases - MongoDB, RethinkDB (relevant for systematization of semi-structured data analyzed by neural networks, such as information for monitoring spatial processes, storage and registration of events); c) graph databases - Neo4j, JanusGraph (useful for storing information about the structure of neural network models); d) columnar databases ClickHouse, Cassandra (represent an uncontested solution for organizing interactive analytical data processing modules (OLAP components) in a neural network repository); e) time series databases - InfluxDB, TimescaleDB (implemented to collect and manage spatial data that change over time, including indicators of the development of natural processes and transactions of the Internet of Things). Thus, for organizing a repository of deep neural network models, it is advisable to use a scientifically grounded set of database management systems integrated into a multi-model storage.

\section{Development of Interfaces for Interacting with the Repository}

The software implementation of the repository was carried out on the basis of artifacts of the object-oriented design stage, including the definition of the use cases of the system, the construction of structural and behavioral UML diagrams with the optimization of the object-oriented metrics of the system. The development of graphical interfaces of the repository was carried out using UI / UX design methodologies using a software stack of web technologies, which will allow you to use the repository from anywhere in the world connected to the Internet. The API is implemented using the REST architectural style. The graphical web interfaces of the deep neural network model repository should be equipped with navigation functions that allow the selection of a relevant machine learning model for solving specific problems of spatial data analysis from any device connected to the Internet (via a web browser). Subsystems for user authentication in the repository system have the task of differentiating the rights to select, use and edit models.

The developed web interfaces solve the problem of providing information about a specific neural network model (including a systematized description, architecture class, format of analyzed and output data, information about topology, subjective and objective performance indicators, recommendations for fine-tuning the model, examples of practice-oriented use). To improve the convenience of interacting with the repository, a subsystem for dynamic visualization of learning models was developed based on adaptive web interfaces in the form of dynamically aligned graphs, with an interactive ability to directly edit the architecture and topology of the neural network model through a thin web client (web browser) (Fig. 2).

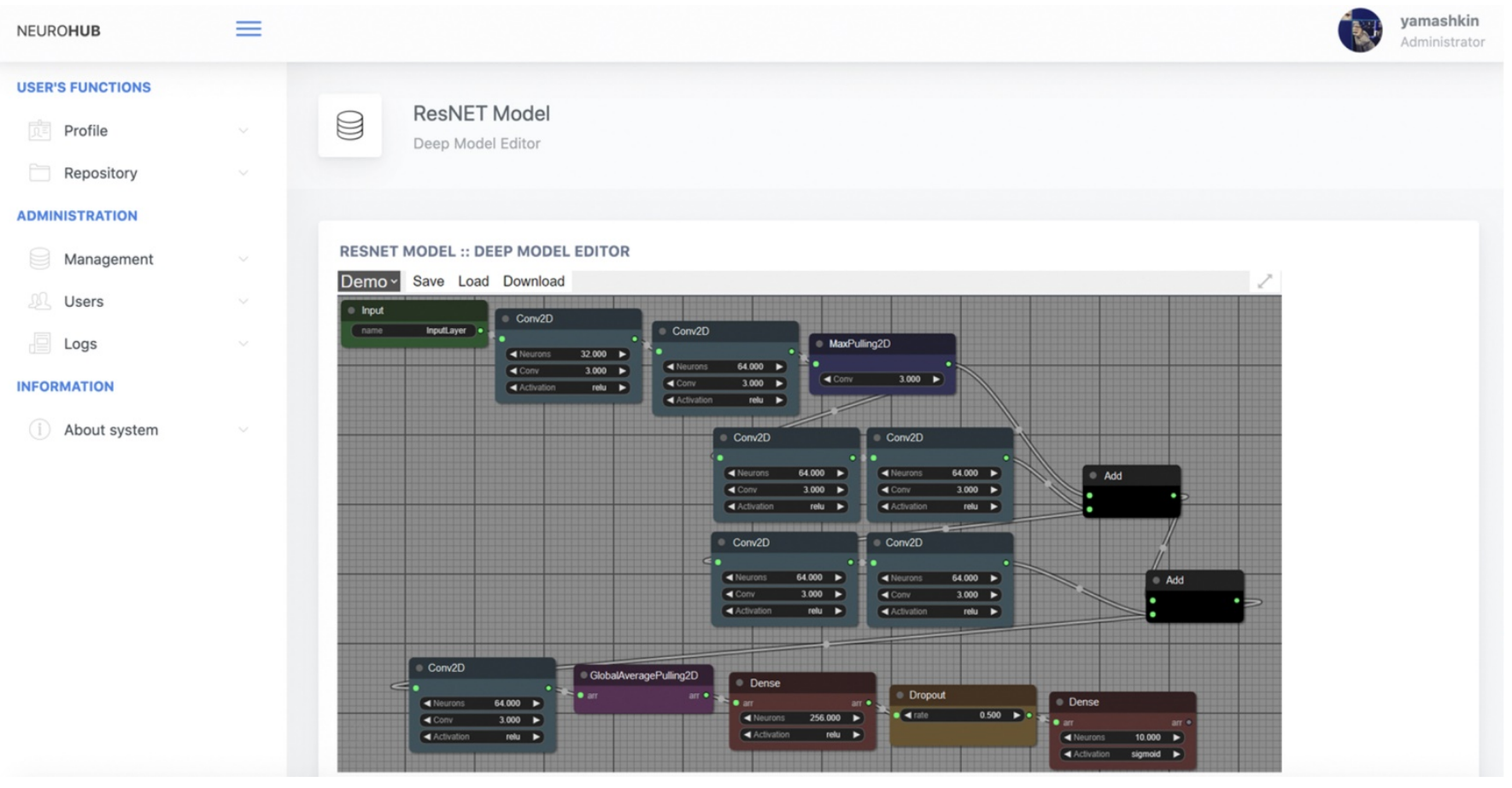

Fig. 2. Graphical Interfaces for Editing a Deep Neural Network Model. 
In the field of neural networks, there are a huge number of libraries, modules and patterns that can be implemented, combined and used for a wide range of tasks. For a visual display of the developed blocks with the aim of their effective use, you can turn to the visual design paradigm. Visual programming is the process of graphically representing a program using a standard set of graphical elements. With visual development, the number of forced control errors in the program is significantly reduced, therefore, the quality of the result obtained is increased.

When developing visual programming languages, drawing of graphs is used as the main approach, that is, in the form of a set of vertices (nodes) connected by arcs (edges). Unlike the textual form of notation, in which objects (symbols and words) form a sequence, and each object is associated only with the left and right "neighbors", the graph form allows you to visually depict more complex relationships, since in it each object can be connected to several others objects. In this sense, the text form is one-dimensional, while the graph form is twodimensional. The ability to vary the geometric dimensions, shape and color of vertices, the appearance and thickness of arcs, change the relative position of the vertices without changing the topology of the graph significantly increases the expressive capabilities of the graph form of the program algorithm.

The creation of a visual (figurative) style of software development is the main motive for the development of graphsymbolic programming (GSP) technology. GSP technology is a technology for designing and coding algorithms and models based on a graphical way of representing programs, with the goal of fully or partially automating the design, coding and testing of software. This programming technology adheres to two fundamental principles: a) visual, graphical form of presentation of program algorithms and other components of their specifications; b) the principle of structured procedural programming. The implementation of this concept is excellent for solving the problem of visual programming of neural networks. Visual programming increases the clarity of the presented codes, significantly reduces the number of errors made at the design and coding stage of programs, and thereby speeds up the development process and increases the reliability of codes of developed programs. Together with the use of the block approach, visual programming methods will not only speed up development due to simple graphical interchangeability of logical blocks, but also provide simple portability of such programs. An application programming interface (API) based on the REST architectural pattern provides a unified interaction with the system for programmatic data exchange, including deep model export, editing, structured data retrieval, and other use cases.

The main directions for the further use of the expected results:

1) Implementation of intelligent systems for forecasting the development of natural and man-made emergency processes based on new technologies of integration, intellectual analysis and dissemination of large geospatial data into the activities of industrial enterprises and executive authorities of the region.
2) Provision of services for the use of a repository of deep neural network models according to the SaaS (Software as a Service) model with the possibility of flexible configuration of the provided solution.

3) Design, development and implementation of geoportal solutions aimed at creating information support for sustainable development of ecological, socio-economic systems of regions, effectively modified for specific corporate customers on the basis of a project-oriented approach. Development and development of socially-oriented geoportal solutions that ensure the effective dissemination of spatial data about nature, economy, social life, history and culture of the regions of the world.

4) Development and implementation of practice-oriented educational programs in the field of sustainable development of regional and global ecological-socio-economic systems with annual bilateral internships and practices to form competencies in the field of information support for sustainable development of world regions and global ecological-socio-economic systems.

The repository of neural networks gains practical value provided that the deep model storage is constantly updated. In the course of further work on the project under a grant from the President of the Russian Federation, it is planned to create and test new models of deep machine analysis, which will be tested in solving specific scientific and practical problems in the field of analyzing the state of natural geosystems, predicting the development and assessing the consequences of emergency situations.

\section{CONCLUSIONS}

The conducted research allows us to determine the reference points for the design, development and implementation of a repository of deep neural network models:

1) Ontological model of the repository, which determines the principles of systematization of deep models for the analysis of spatial data according to the classes of problems to be solved, the nature and dimension of the analyzed data, architecture and topology, properties of efficiency.

2) A formalized storage scheme for deep machine analysis models of spatial data in the form of a meta-language that allows them to be converted into representations used by modern machine learning software systems (Caffe, Torch, MXNet, TensorFlow, Keras).

3) Adaptive graphical web interfaces of the repository with navigation functions that allow you to select the relevant machine learning model for solving specific problems of spatial data analysis.

4) Subsystem for user authentication in the repository in order to restrict the rights to read, use and edit neural network models.

5) Adaptive web interfaces for obtaining systematized information about the required deep neural network model, including a systematized description, objective and subjective performance indicators, type of architecture and topological 
scheme (branching and chain structures of layers of various types: fully connected, convolutional, recurrent), practical recommendations for flexible setting hyper-parameters of the model, examples of applied use.

6) Subsystem for visualization of the neural network model of deep learning in the form of a graph diagram, with the possibility of interactive online editing of the topology and architecture of the model through a web browser, a thin client.

7) Recommended web system for the selection and configuration of deep neural network models, which allows to provide relevant search and fine-tuning for solving specific design problems in the field of spatial data analysis.

8) An application programming interface based on the REST architectural style, which allows for a unified interaction for programmatic data exchange with the repository (including import and export of deep models, obtaining information about them).

9) Database of deep neural network models repository, which includes pretrained models based on convolutional and recurrent layers, regularization, normalization and subdescritization modules with the possibility of their direct use for solving applied problems or further fine tuning.

The implementation of the project will make it possible to form a platform solution for solving urgent scientific problems of consolidation, storage, selection and effective use of deep models for solving project-oriented problems in the field of analyzing large arrays of spatial data. Integration of neural network models into the repository will allow starting the formation of a bank of intelligent algorithms designed to solve specific problems in the field of spatial data analysis to ensure sustainable development of regions, but also to solve the problem of interactive search for an effective model by forming a system of recommendations and developing expert tools that optimize the choice of algorithms.

\section{ACKNOWLEDGMENT}

This work was supported by Grant of the President of the Russian Federation under Project no. MK-199.2021.1.6.

\section{REFERENCES}

[1] S. Yamashkin, M. Radovanovic, A. Yamashkin, and D. Vucovic, "Using Ensemble Systems to Study Natural Processes," Journal of Hydroinformatics, vol. 20, no. 4, pp. 753-765, Apr. 2018.

[2] R. A. Schowengerdt, Remote sensing: models and methods for image processing, 3rd ed. Orlando, FL, USA: Academic Press, 2006, pp. 387456.

[3] X. X. Zhu, D. Tuia, L. Mou, G. S. Xia, L. Zhang, F. Xu, and F. Fraundorfer, "Deep learning in remote sensing: A comprehensive review and list of resources,” IEEE Geoscience and Remote Sensing
Magazine, vol. 5, no. 4, pp. 8-36, Oct. 2017, DOI. 10.1109/MGRS.2017.2762307.

[4] L. Zhang, L. Zhang, and B. Du, "Deep learning for remote sensing data: A technical tutorial on the state of the art," IEEE Geoscience and Remote Sensing Magazine, vol. 4, no. 2, pp. 22-40, Jun. 2016, DOI. 10.1109/MGRS.2016.2540798.

[5] S. V. Kovshov, and S. Tingnting, “Application of Computer Modeling for the Accident Rate Assessment on Separate Sites of the MoheDaqing Oil Pipeline in Permafrost Conditions," Transportation Infrastructure Geotechnology, vol. 7, pp. 605-617, Jul. 2020.

[6] C. Tao, H. Pan, Y. Li, and Z. Zou, "Unsupervised spectral-spatial feature learning with stacked sparse autoencoder for hyperspectral imagery classification,” IEEE Geoscience and Remote Sensing Let., vol. 12, no. 12, pp. 2438-2442, Dec. 2015, DOI. 10.1109/LGRS.2015.2482520.

[7] Y. LeCun, Y. Bengio, and G. Hinton, “Deep learning,” Nature, vol. 521, no. 7553, pp. 436, May 2015, DOI. 10.1038/nature14539.

[8] W. Li, H. Liu, Y. Wang, Z. Li, Y. Jia, and G. Gui “Deep learning-based classification methods for remote sensing images in urban built-up areas,” IEEE Access, no. 7, 36274-36284, Mar. 2019, DOI. 10.1109/ACCESS.2019.2903127.

[9] H. Wu and S. Prasad, "Convolutional recurrent neural networks for hyperspectral data classification,” Remote Sensing, vol. 9, no. 3, 298, Mar. 2017, DOI. 10.3390/rs9030298.

[10] W. Li, H. Fu, L. Yu, P. Gong, D. Feng, C. Li, and N. Clinton, “Stacked Autoencoder-based deep learning for remote-sensing image classification: a case study of African land-cover mapping," International journal of remote sensing, vol. 37, no. 23, pp. 5632-5646, Dec. 2016, DOI. 10.1080/01431161.2016.1246775.

[11] M. Abadi, P. Barham, J. Chen, Z. Chen, A. Davis, J. Dean, and X. Zheng, "Tensorflow: A system for large-scale machine learning," 12th \{USENIX\} symposium on operating systems design and implementation, pp. 265-283, 2016.

[12] A. Paszke, S. Gross, F. Massa, A. Lerer, J. Bradbury, G. Chanan, S. Chintala, "Pytorch: An imperative style, high-performance deep learning library,” arXiv preprint arXiv:1912.01703, 2019.

[13] J. V. Alva, “Neural Network Framework,” Beginning Mathematica and Wolfram for Data Science, pp. 375-406, 2021.

[14] Amazon Web Services Marketplace - Machine Learning. Accessed: May. 2021. [Online]. Available: https://aws.amazon.com/marketplace/ solutions/machinelearning.

[15] Wolfram Repository of Neural Network Models, Apr. 2021, [online] Available: http://resources.wolframcloud.com/NeuralNetRepository.

[16] D. Powers, "Evaluation: From Precision, Recall and F-Measure to ROC, Informedness, Markedness \& Correlation," Journal of Machine Learning Technologies, vol. 2, no. 1, pp. 37-63, Jan. 2011, DOI. 10.9735/2229-3981.

[17] E. Yamashkina, S. Kovalenko, and O. Platonova, "Development of repository of deep neural networks for the analysis of geospatial data," IOP Conference Series: Materials Science and Engineering, vol. 1047, no. 1, 012124, Feb. 2021.

[18] S. Ioffe and C. Szegedy, "Batch normalization: Accelerating deep network training by reducing internal covariate shift," arXiv: 1502.03167 [cs.LG], Mar. 2015.

[19] M. Xiu, Z. M. J. Jiang, B. Adams, “An Exploratory Study on MachineLearning Model Stores,” arXiv preprint, 1905.10677, 2020. 